\title{
A New DCT based Channel Estimation Algorithm for OFDM Systems
}

\author{
Antony Nycil P.A. \\ Dept. of Electronics and Communication \\ FISAT \\ M.G. University
}

\author{
Anoop E G \\ Asst. Professor \\ Dept. of Electronics and Communication \\ FISAT, M.G. University
}

\begin{abstract}
This paper deals with the estimation of the channel in orthogonal frequency division multiplexed systems. In this paper, a new DCT-based pilot-symbol-aided channel estimation method is proposed. The energy leakage in multipath channel with non-sample-spaced time delays is eliminated here. The proposed method uses symmetric property to extend the LS estimate in frequency domain to remove the effects of non-sample spaced time delays. The computer simulation results demonstrate that the new method can reduce the leakage energy efficiently, and the performance of the new channel estimation method is better than the LS and improved DFT algorithm.
\end{abstract}

\section{Keywords}

Channel estimation; OFDM; energy leakage; improved DFT; DCT.

\section{INTRODUCTION}

Orthogonal frequency division multiplexing (OFDM) is a technique in which a large number of narrowband sub channels are transmitted in parallel over a large bandwidth thereby increasing the symbol duration and reducing the ISI. OFDM is an effective technique for combating multipath fading and for high-bit-rate transmission over mobile wireless channels. OFDM has been adopted in several wireless standards such as digital audio broadcasting (DAB), digital video broadcasting (DVB-T), the IEEE 802.11a local area network (LAN) standard and the IEEE 802.16a metropolitan area network (MAN) standard.

In order to achieve the potential advantages of OFDM-based systems, the channel coefficients should be estimated with minimum error. Various OFDM channel estimation schemes have been proposed in the literature. The pilot aided channel estimation helps to increase data rate. Least squares (LS) approach and Minimum Mean Square Error (MMSE) estimation is introduced in [1,2]. The LS estimation is the simplest channel estimation method. This algorithm has low complexity. For MMSE computational complexity is higher but result is better than LS estimation. The different interpolation methods used in pilot aided channel estimation are mentioned in $[2,3]$.

In this paper a new DCT based channel estimation algorithm is introduced. This algorithm can make good compromise between performance and computational complexity. In non sample-spaced multipath channels, the channel impulse response will leak to all taps in the time domain. The improved DFT based [4] and DCT based [5,6] channel estimation methods are the two published methods used for the channel estimation in the case of non-sample spaced time delays. The improved DFT based channel estimation method uses a symmetric extension in frequency domain [7] to reduce the leakage energy problem. The performance of the improved DFT based method degrades when more noise exists. The DCT based method helps to reduce the noise component and the high frequency components in the transform domain $[8,9]$. Based on these two methods, a new method to solve the problem of energy leakage is proposed.

The outline of the paper is as follows. In Section 2, OFDM system model is described. In Section 3, LS algorithm is introduced. The new method is explained in Section 4. Simulation results are presented in Section 5. Finally Section 6 provides the conclusions.

\section{OFDM SYSTEM MODEL}

\subsection{Multipath channel model}

The channel impulse response $h(t)$ is treated as a time limited pulse train and the channel impulse response in multipath fading channel could be given by

$$
h(t)=\sum_{m=0}^{M-1}\left(a_{m} \delta\left(\mathrm{t}-\tau_{\mathrm{m}} \mathrm{T}_{\mathrm{s}}\right)\right.
$$

where $\mathrm{M}$ is the total number of multipaths, the amplitude $a_{m}$ are complex valued channel coefficient of the mth path, $\mathrm{T}_{\mathrm{s}}$ is sampling interval and $\tau_{\mathrm{m}} \mathrm{T}_{\mathrm{s}}$ is delay of mth path. If $\tau_{\mathrm{m}}$ is an integer the multipath channel is sample spaced channel and when $\tau_{\mathrm{m}}$ is not an integer the multipath channel is nonsample spaced channel. In this work the non-sample spaced channel is considered. After sampling the frequency response of $h(t)$ the channel impulse response can be given by[10]

$$
h_{n}=\frac{1}{\mathrm{~N}} \sum_{m=1}^{M}\left(a_{m} \mathrm{e}^{-\mathrm{j} \frac{\pi}{\mathrm{N}}\left(\mathrm{n}+(\mathrm{N}-1) \tau_{\mathrm{m}}\right)} \frac{\sin \left(\pi \tau_{\mathrm{m}}\right)}{\sin \left(\frac{\pi}{\mathrm{N}}\left(\tau_{\mathrm{m}}-\mathrm{n}\right)\right)}\right)
$$

The channel impulse response in frequency domain can be given by

$H(k)=\sum_{m=1}^{M} h_{n} \mathrm{e}^{\left(-\mathrm{j} 2 \pi \frac{\mathrm{km}}{\mathrm{N}}\right)}$ 


\subsection{OFDM model}

In the case of OFDM the data stream is modulated by inverse fast Fourier transform (IFFT) at the sending end and a guard interval is added for every OFDM symbol to eliminate ISI caused by multi-path fading channel. The receiver performs opposite operations. The received signal can be expressed as

$$
\mathrm{Y}_{\mathrm{l}}[\mathrm{k}]=\mathrm{H}_{\mathrm{l}}[\mathrm{k}] \mathrm{X}_{\mathrm{l}}[\mathrm{k}]+\mathrm{Z}_{\mathrm{l}}[\mathrm{k}]
$$

Where $\mathrm{X}_{1}[\mathrm{k}], \mathrm{Y}_{1}[\mathrm{k}], \mathrm{H}_{1}[\mathrm{k}]$, and $\mathrm{Z}_{1}[\mathrm{k}]$ denote the kth subcarrier frequency components of the lth transmitted symbol, received symbol, channel frequency response, and noise in the frequency domain, respectively.

\section{CHANNEL ESTIMATION ALGORITHM}

The simplest of the channel estimation algorithms is the LS estimation algorithm. So LS algorithm is described first.

\subsection{LS Channel Estimation}

In the simplest case the channel estimates are found by straightforward multiplying the received pilot subsymbols by the inverse of the reference pilot subsymbol values - the socalled frequency-domain least squares (LS) estimator, which can be written as

$\widehat{\mathrm{H}}_{\mathrm{p}}^{\mathrm{LS}}=\mathrm{X}_{\mathrm{p}}{ }^{-1} \mathrm{Y}_{\mathrm{p}}=\left[\mathrm{X}_{p_{0}}{ }^{-1} \mathrm{Y}_{p_{0}} \quad \ldots \ldots \ldots \mathrm{X}_{\mathrm{p}_{\mathrm{p}-1}}{ }^{-1} \mathrm{Y}_{\mathrm{p}_{\mathrm{p}-1}}\right]^{\mathrm{T}}$

Where $\left(p_{0} \ldots \ldots . p_{p-1}\right)$ denotes the set of subcarriers, which are used to carry pilot subsymbols.

After that, the channel estimates $\widehat{\mathrm{H}}_{\mathrm{pz}}^{\mathrm{LS}}, z=0, \ldots, P-1$,obtained at the pilot positions, are interpolated over the whole band to obtain the LS estimate $\widehat{H}_{L S}(k)$.

\section{A NEW DCT BASED CHANEL ESTIMATION METHOD}

Step1: Calculate the LS estimate $\widehat{H}_{L S}(k)$

Step2: Extend $\widehat{H}_{L S}(k)$ with a symmetric signal of its own:

$$
\widehat{H}_{\text {symmetric }}(k)=\left\{\begin{array}{cc}
\widehat{H}_{L S}(k) & 0 \leq k \leq N-1 \\
\widehat{H}_{L S}(2 N-1-k) & N \leq k \leq 2 N-1
\end{array}\right.
$$

Step 3: Covert $\widehat{H}_{\text {symmetric }}(k)$ to cosine domain by DCT. The channel impulse response in transform domain is then given by

$$
\widehat{H}_{\text {symmetric }}(n)^{D C T}=D C T\left[\widehat{H}_{\text {symmetric }}(k)\right]
$$

Step4: The channel response in the transform domain can be expressed by:

$\widehat{H}_{D C T}(n)=\left\{\begin{array}{cc}0 & l_{\text {leak }} \leq n \leq 2 N-1-l_{\text {leak }} \\ \widehat{H}_{\text {symmetric }}(n)^{D C T} & \text { other }\end{array}\right.$

Where $l_{\text {leak }}$ is assumed as the first path number of starting leaking energy. The performance improves as $l_{\text {leak }}$ increases.

Step 5: After padding with zeros, convert $\widehat{H}_{D C T}(n)$ back to frequency domain by IDCT

$$
\widetilde{H}(k)=\operatorname{IDCT}\left[\widehat{H}_{D C T}(n)\right] \quad 0 \leq k \leq 2 N-1
$$

Step 6: According to the symmetric property, the corresponding frequency response is expressed as:

$\widetilde{H}(k)=\frac{\widetilde{H}(k)+\widetilde{H}(2 N-1-k)}{2} \quad 0 \leq k \leq N-1$

\section{SIMULATION RESULTS}

The performances of the existing and proposed methods are investigated through MATLAB simulation. An OFDMA system with $N=64$ subcarriers is considered. The guard interval is 24. A 4-path channel model is used. The symbols are modulated by 16QAM.The delay of the antenna is delay $=(0,1.5,2.5,3.5,4.5) \mu \mathrm{s}$. Fig. 1 shows the least squares estimation using linear and cubic spline interpolation methods. Fig.2 shows the estimated channel using conventional DCT based channel estimation. Using the estimated channel the data transmitted is extracted and the number of points at which the estimated data differed from the original transmitted data for 10000 iterations is calculated and the result is shown in table1. From table 1it can see that cubic spline interpolation method is better than the liner interpolation method and hence cubic spline interpolation method is used in further calculations. From table1 it can also been seen that when the DCT based method is used a better result obtained. Fig. 3 shows the estimated channel using the improved DFT based method and table 2 shows the number of points at which the estimated data differ from the original transmitted data for 10000 iterations for the improved DFT based method. From table 2, it can see that as the leakage path increases the result of improved DFT based method becomes better. Fig. 4 shows the estimated channel using the new DCT based method and corresponding result is shown in table 3 which shows that for the same leakage path the new DCT based method is better than the improved DFT based method. Also it can be seen that as the leakage path number increases the performance of the new DCT based method gets improved. So proper $1_{\text {leak }}$ should be selected in order to achieve better performance. From tables 1, $2 \& 3$ and figures 1 , $2,3 \& 4$ it can see that the new DCT based method performs better.

$$
0 \leq n \leq 2 N-1
$$




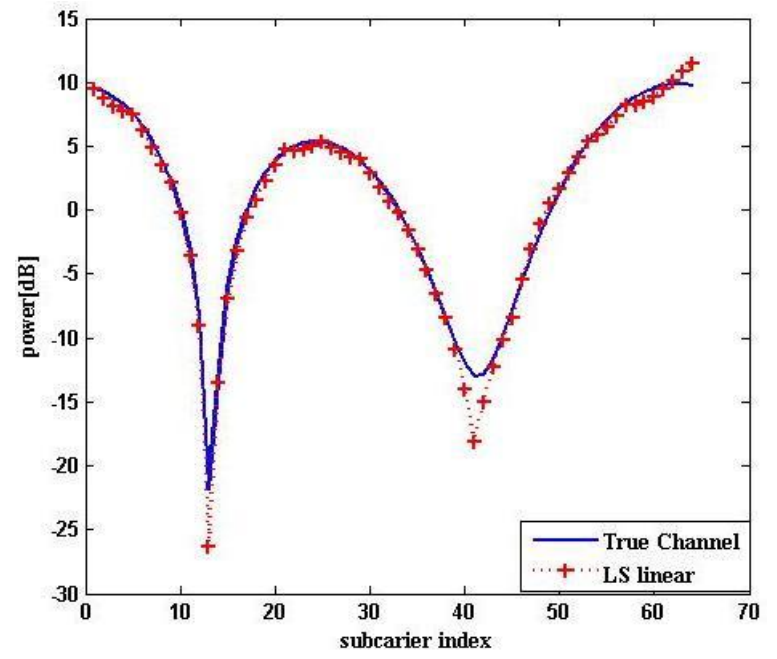

(a)

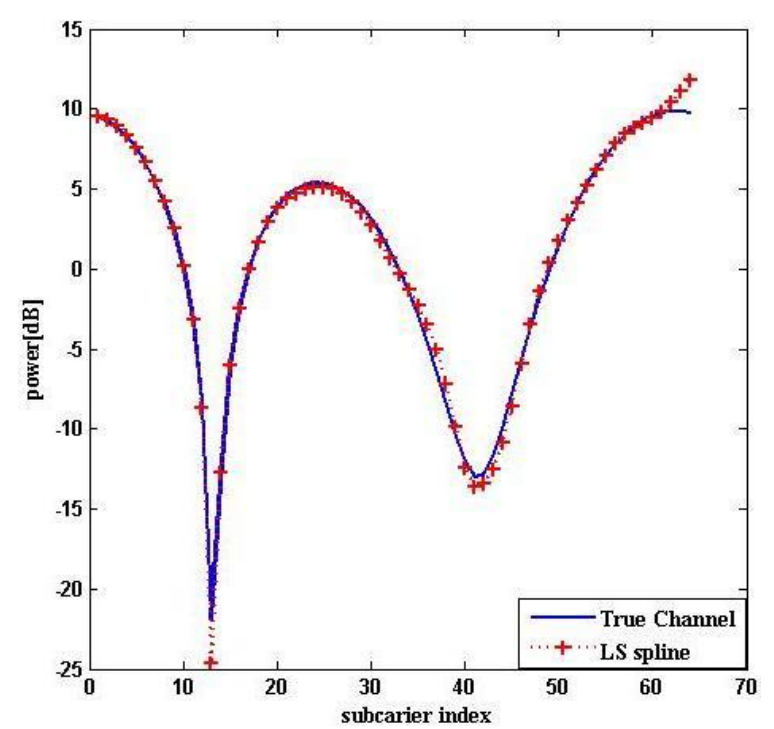

(b)

Fig1.Plot between channel power and subcarrier index for (a) LS-linear (b) LS-cubic Spline

Table.1 comparison between orginal data transmitted and estimated data for 10000 iteration

\begin{tabular}{|c|c|}
\hline System & $\begin{array}{c}\text { Number of points at which estimated data differ } \\
\text { from transmitted data for 10000 iteration }\end{array}$ \\
\hline LS-linear & 28877 \\
\hline LS spline & 11335 \\
\hline DCT & 11330 \\
\hline
\end{tabular}

Table2.comparison between original data transmitted and estimated data for 10000 iterations

\begin{tabular}{|c|c|c|}
\hline & $1_{\text {leak }}$ & $\begin{array}{c}\text { Number of points at which } \\
\text { estimated data differ from } \\
\text { transmitted data for 10000 } \\
\text { iteration }\end{array}$ \\
\hline Improved DFT-case1 & 45 & 11358 \\
\hline Improved DFT-case2 & 59 & 11350 \\
\hline Improved DFT-case3 & 60 & 11339 \\
\hline
\end{tabular}

Table 3.comparison between original data transmitted and estimated data for 10000 iterations

\begin{tabular}{|c|c|c|}
\hline & $1_{\text {leak }}$ & $\begin{array}{c}\text { Number of points at which } \\
\text { estimated data differ from } \\
\text { transmitted data for 10000 } \\
\text { iteration }\end{array}$ \\
\hline NEW DCT-case1 & 45 & 11319 \\
\hline NEW DCT-case2 & 59 & 11299 \\
\hline NEW DCT-case3 & 60 & 11215 \\
\hline
\end{tabular}

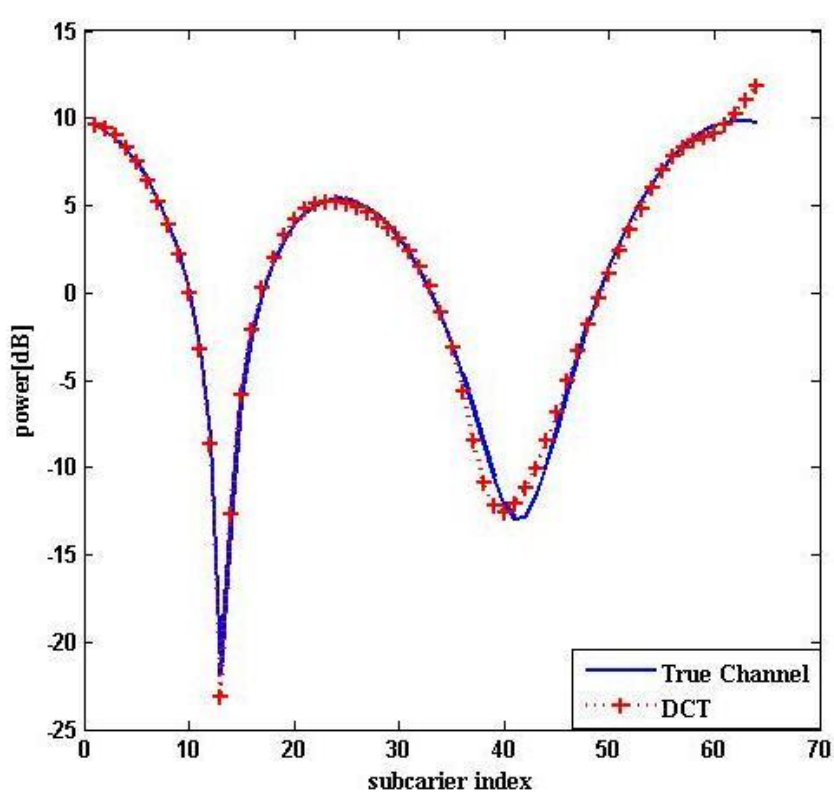

Fig2. Plot between channel power and subcarrier index for conventional DCT based method 


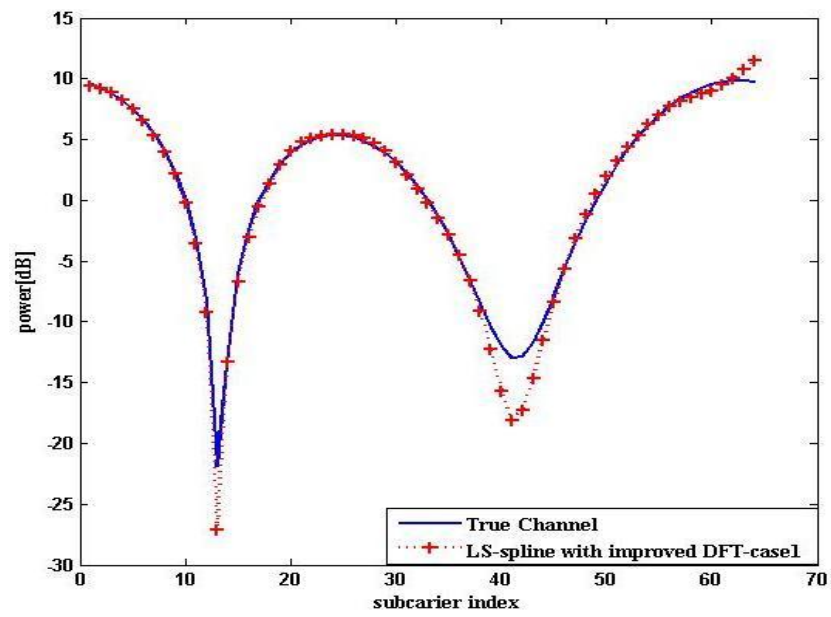

(a)

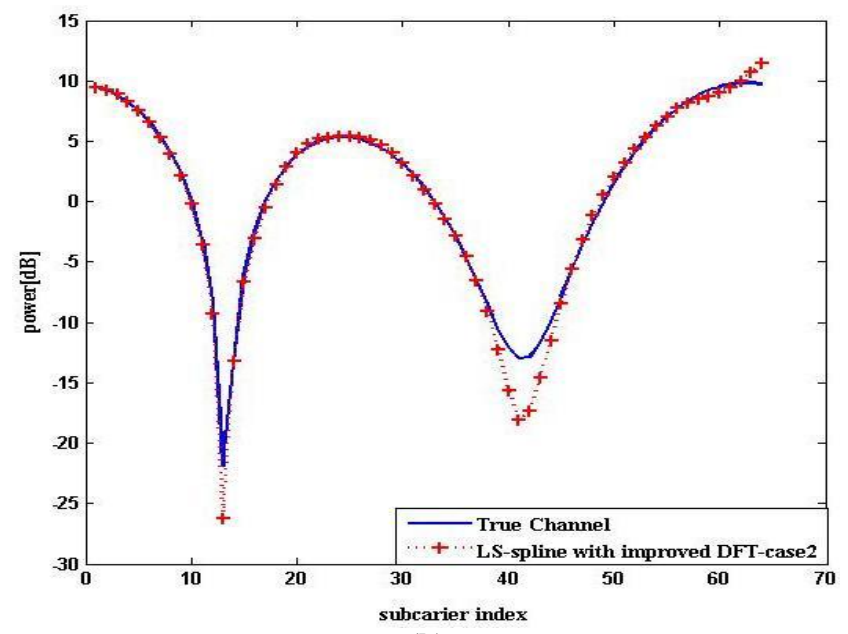

(b)

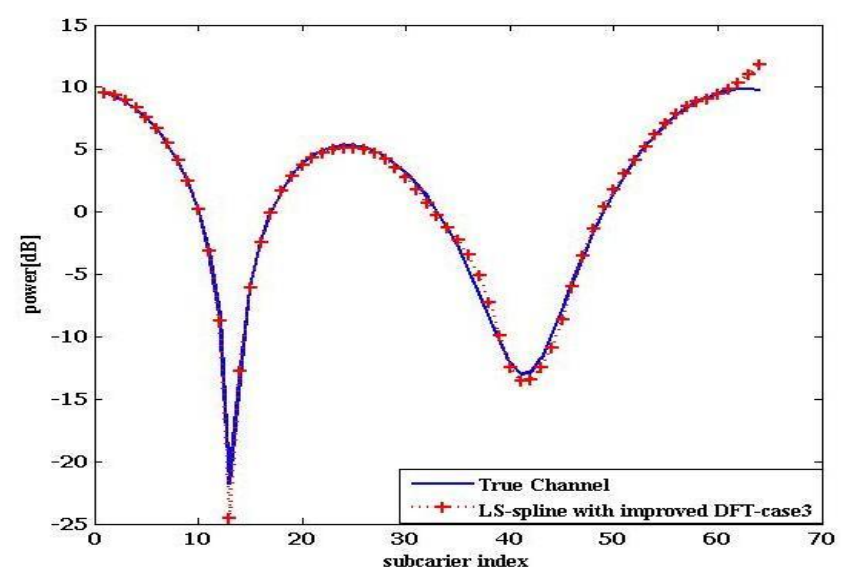

(c)

Fig3 Plot between channel power and subcarrier index for

(a) LS-Spline with improved DFT for $l_{\text {leak }}=45$

(b) LS-Spline with improved DFT for $l_{\text {leak }}=59$

(c) LS-Spline with improved DFT for $l_{\text {leak }}=60$

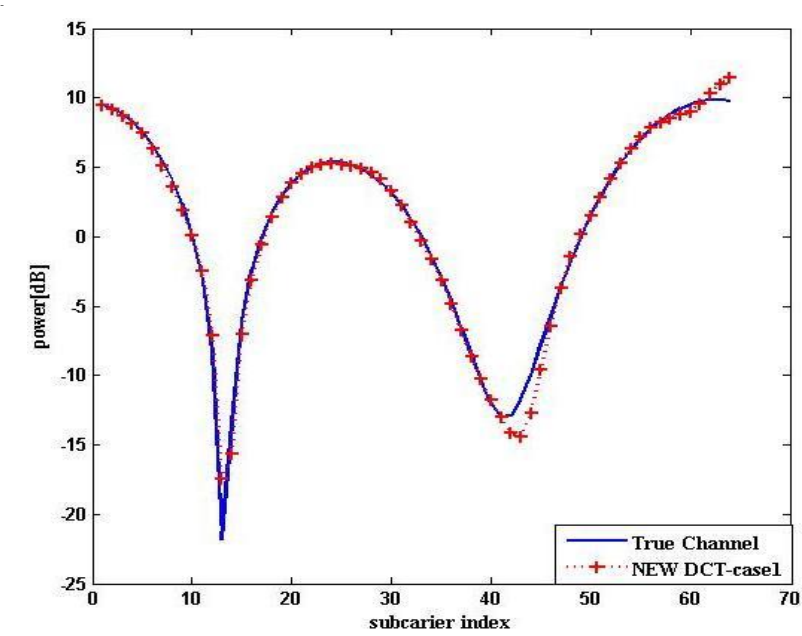

(a)

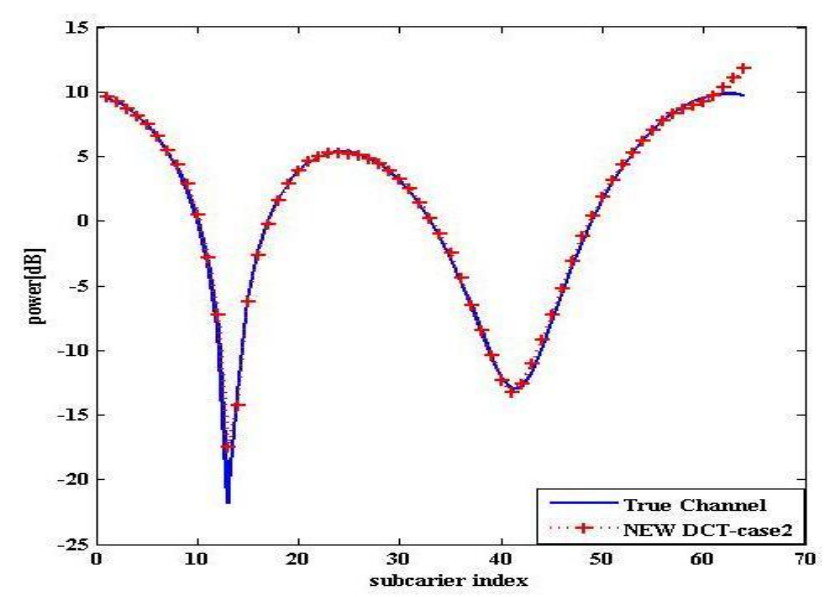

(b)

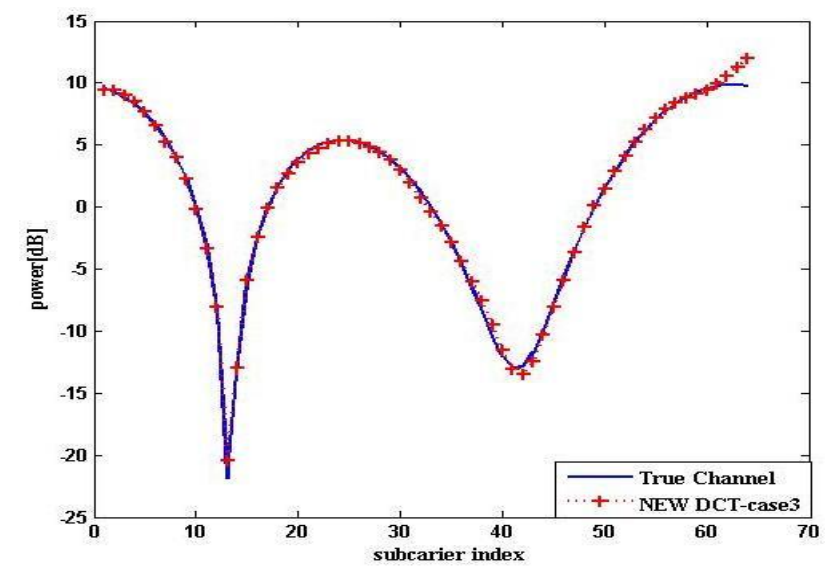

(c)

Fig4 Plot between channel power and subcarrier index for (a) LS-Spline with new DCT for $l_{\text {leak }}=45$

(b) LS- Spline with new DCT for $l_{\text {leak }}=59$

(c) LS-Spline with new DCT for $l_{\text {leak }}=60$ 


\section{CONCLUSION}

A new DCT-based channel estimation method with symmetric extension is proposed in this paper. The new method uses the symmetric property and cosine domain operations to reduce the effect of leakage power in the case of non-sample spaced multipath channels. The existing methods of channel estimation for non-sample spaced multipath channels i.e. improved DFT based channel estimation method and DCT based channel estimation method are simulated using MATLAB. The Simulation results show that the new DCT based method is better than the existing methods like LS estimation, improved DFT based estimation and conventional DCT based channel estimation methods. If the noise components are further reduced the performance of the estimator will improve. So further research is needed to optimize the method to reduce the noise components to get an improved result.

\section{REFERENCES}

[1] Sinem Coleri, Mustafa Ergen, Anuj Puri, and Ahmad Bahai, "Channel Estimation Techniques Based on Pilot Arrangement in OFDM Systems," IEEE transactions on broadcasting, vol. 48, no. 3, september 2002.

[2] Sinem Coleri, Mustafa Ergen,Anuj Puri, Ahmad Bahai, "A Study of Channel Estimation in OFDM Systems," Vehicular Technology Conference, 2002. Proceedings. VTC 2002-Fall. 2002 IEEE 56 ${ }^{\text {th }}$, Page(s): 894 - 898 vol.2.

[3] Xiaodai Dong, Wu-Sheng Lu, Anthony C. K. "Linear Interpolation in Pilot Symbol Assisted Channel Estimation for OFDM" IEEE transactions on wireless communications, VOL. 6, NO. 5, MAY 2007
[4] Zhang Jie, Huang Liqun, "An Improved DFT-based Channel Estimation Algorithm For MIMO-OFDM Systems," Consumer Electronics, Communications and Networks (CECNet), 2011 International Conference on Page(s): 3929 - 3932.

[5] Saqib Saleem, Qamar-ul-Islam, "On Comparison of DFT-Based and DCT-Based Channel Estimation for OFDM System," IJCSI International Journal of Computer Science Issues, Vol. 8, Issue 3, No. 2, May 2011.

[6] Moussa Diallo, Rodrigue Rabineau, Laurent Cariou, Maryline Hélard. "Transform Domain based Channel Estimation for 3GPP/LTE Systems" Communications and Networking September 2010.

[7] Y. Wang, L. Li, P. Zhang and Z. Liu, "Channel estimation for OFDM systems in non-sample-spaced multipath channels," Electronics Letters, vol. 45, January. 2009.

[8] H. Kobayaki and k. Mori. "Proposal of OFDM channel estimation method using discrete cosine transform". Personal, Indoor and Mobile Radio Communications, 2004. Vol.3 .Page(s):1797-1801.

[9] T. Daisuke, T. Yasuhiko, and S. Kasumi. "Channel Estimation Scheme with low complexity Discrete Cosine Transformation in MIMO-OFDM System". Vehicular Technology Conference, 2007. Page(s): 486 - 490.

[10] Jan-Jaap van de Beek, O.Edfors, and M.Sandell, "On channel estimation in OFDM systems," Presented at in proceedings of Vehicular Technology, Chicago, pp. 815819, 1995. 